\title{
PERMUTATIONAL PRODUCTS AND REGULAR PRODUCTS OF GROUPS
}

\author{
BY \\ R. B. J. T. ALLENBY
}

1. Introduction. In [3] Gregorac asked what relations hold between the permutational products (see $\S 2$ ) of an amalgam $(A, B ; H)$ of groups $A, B$ with the amalgamated subgroup $H$ and the generalised regular products (see \$2) of the amalgam.

At the outset it is clear that if $G$ is a generalised regular product of $A$ and $B$ amalgamating $H$, it is by no means necessary that $G$ is also a permutational product of $A$ and $B$ amalgamating $H$. For, if $A$ and $B$ are finite groups, every permutational product of $(A, B ; H)$ must also be finite, whereas the generalised free product of $(A, B ; H)$ is a generalised regular product (see Definition 2.8) and is, of course, an infinite group.

However, in the other direction, we can prove

THEOREM. Let $G$ be a permutational product of $(A, B ; H)$. Then $G$ is a generalised regular product of $(A, B ; H)$.

This will be proved in $\S 3$, following preliminaries in $\S 2$.

2. Notation and preliminaries. The notation will be that usually employed in group-theoretical writings. We mention, however, the following. If $G$ is a group and $X$ a subgroup, $X^{G}$ will denote the normal closure of $X$ in $G$. If $X, Y$ are subgroups of $G[X, Y]$ will denote the subgroup of $G$ generated by the set of all elements $[x, y]=x^{-1} y^{-1} x y$ where $x \in X, y \in Y$. In the case where $G$ is generated by subgroups $A_{\alpha}(\alpha \in I)$, the cartesian $\left[A_{\alpha}\right]^{G}$ is the normal closure in $G$ of the subgroup generated by all $\left[x_{\alpha}, x_{\beta}\right]$ where $\alpha, \beta \in I, \alpha \neq \beta$. By $\left\{{ }_{\alpha} A\right\}$ we shall mean the subgroup generated by all the $A_{\beta}(\beta \in I, \beta \neq \alpha)$. It is clear that $\left[A_{\alpha}\right]^{G} \leqq\left\{{ }_{\alpha} A\right\}^{G}$ for any given $\alpha \in I$.

We shall require the following results.

LEMMA 2.1 [2]. If $G$ is generated by subgroups $X$ and $Y$, then $[X, Y] \triangleleft G$. In particular, $[G, X] \triangleleft G$ for any subgroup $X$ of $G$.

Lemma $2.2[2] . X^{G}=X[X, G]$ for any subgroup $X$ of $G$.

LEMMA 2.3 [2]. If $\phi$ is a homomorphism of $G$, we have $[X, Y] \phi=[X \phi, Y \phi]$ and $\left(\left[A_{\alpha}\right]^{G}\right) \phi=\left[A_{\alpha} \phi\right]^{G \phi}$ for subgroups $A_{\alpha}$ in $G$.

Received by the editors August 24, 1967. 
Definition 2.4. An amalgam $(A, B ; H)$ of two groups $A$ and $B$ with the amalgamated subgroup $H$ is simply their set-theoretical union $A \cup B$ where $A \cap B=H$.

Definition 2.5 [4]. A normal subamalgam of $(A, B ; H)$ is an amalgam $(X, Y ; K)$ where $X \triangleleft A, Y \triangleleft B$ and $X \cap H=Y \cap H=K$. One can then form the factor amalgam $(A / X, B / Y ; H / K)$ where $H X / X$ and $H Y / Y$ are identified via their natural isomorphisms with $H /(H \cap X)=H /(H \cap Y)=H / K$.

We now come to the definition of permutational products (see [6]).

Let $(A, B ; H)$ be an amalgam and choose arbitrary (but fixed) systems of left coset representatives $S, T$ of $A, B$ respectively modulo $H$. Form the set $W$ of all triplets $(s, t, h)$ where $s \in S, t \in T, h \in H$. For arbitrary $a \in A$ define a map $\rho(a)$ of $W$ into itself by the rule $(s, t, h)^{\rho(a)}=\left(s_{1}, t, h_{1}\right)$ where $s_{1} \in S, h_{1} \in H$ and $s_{1} h_{1}=s h a$.

Similarly, if $b \in B$ we define $(s, t, h)^{\rho(b)}=\left(s, t_{1}, h_{2}\right)$ where $t_{1} \in T, h_{2} \in H$ and $t_{1} h_{2}=t h b$.

It was shown in [6] that:

(i) If $h^{0} \in H$, then there is no ambiguity in the definition of $\rho\left(h^{0}\right)$.

(ii) $\rho(a), \rho(b)$ are permutations of $W$.

(iii) The set $\rho(A)$ of all $\rho(a)$ is a group of permutations isomorphic with $A$; the set $\rho(B)$ of all $\rho(b)$ is a group isomorphic with $B$.

(iv) $\rho(A) \cap \rho(B)=\rho(H)$ is isomorphic with $H$.

Definiton 2.6. The permutational product of $(A, B ; H)$ with systems of coset represesentatives $S, T$ is the subgroup of the symmetric group on $W$ generated by $\rho(A), \rho(B)$. (Distinct pairs of systems in general give rise to nonisomorphic groups, see [6].)

We now require

THEOREM 2.7 [7]. Let $F$ be the generalised free product of its subgroups $A_{\alpha}(\alpha \in I)$ with amalgamations $A_{\alpha} \cap A_{\beta}=H_{\alpha \beta}(\alpha \neq \beta)$, and let $G$ be a group containing homomorphic image $A_{\alpha} \phi_{\alpha}$ of the $A_{\alpha}$ where two mappings $\phi_{\alpha}$, $\phi_{\beta}$ agree on their common region $H_{\alpha \beta}$ of definition. Then the $\phi_{\alpha}$ can be simultaneously extended to a unique homomorphism $\phi$ of the whole of $F$ into $G$.

This enables us to give

Definition 2.8 [8]. Let $G$ be any group generated by subgroups $A_{\alpha}(\alpha \in I)$ with amalgamations $A_{\alpha} \cap A_{\beta}=H_{\alpha \beta}$ and suppose $F$ is the free product of the $A_{\alpha}$ with these amalgamations. Then by Theorem 2.7 , there is a unique (natural) homomorphism $\phi$ from $F$ to $G$ which extends the isomorphisms from the $A_{\alpha}$ in $F$ onto the $A_{\alpha}$ in $G$. If the kernel of $\phi$ is contained in $\left[A_{\alpha}\right]^{F}, G$ is called a generalised regular product of the $A_{\alpha}$ with amalgamations $H_{\alpha \beta}$.

Clearly every generalised free product is a generalised regular product of the same subgroups with the same amalgamations.

Finally we give

LEMMA 2.9 [8]. Let $G$ be a generalised regular product of its subgroups $A_{\alpha}(\alpha \in I)$ with the single central amalgamated subgroup $H$. Then, for each $\alpha \in I, A_{\alpha} \cap\left[A_{\alpha}\right]^{G}=E$. 
LEMMA 2.10 [1]. If $G$ is a generalised regular product of its subgroups $A_{\alpha}(\alpha \in I)$ with its amalgamated subgroup $H$, then $A_{\alpha} \cap\left\{{ }_{\alpha} A\right\}^{G}=H^{A_{\alpha}}$.

LEMMA 2.11 [5]. If $\phi$ is a homomorphism of a group $G$ and if $X$ and $Y$ are subgroups such that $Y$ contains the kernel of $\phi$, then $(X \cap Y) \phi=X \phi \cap Y \phi$.

\section{Proof of theorem.}

Lemma 3.1. Let $F$ be the free product of $\mathscr{A}=(A, B ; H)$. Then, in $F, H \cap[A, B]$ $=(H \cap[A, H])(H \cap[H, B])$.

Proof. By Lemma 2.1, $[A, H] \triangleleft A$ and $[B, H] \triangleleft B$. It follows that $H \cap[A, H]$ and $H \cap[H, B]$ are normal subgroups of $H$ and hence that they commute. Set $N=(H \cap[A, H])(H \cap[H, B])$ and consider the subgroups $[A, H] N$ and $[H, B] N$ of $A$ and $B$, respectively. Using the above remarks it is easy to check that $[A, H] N \triangleleft A$ and $[H, B] N \triangleleft B$ and that $[A, H] N \cap H=[H, B] N \cap H=N$. By Definition 2.5 we can therefore construct the amalgams $\mathscr{B}=(X, Y ; N)$ and $\mathscr{C}=(A / X, B / Y ; H / N)$ where $X=[A, H] N$ and $Y=[H, B] N$.

Let the free product of $\mathscr{C}$ be denoted by $\bar{F}$. Now $\bar{F}$ is generated by homomorphic images of $A$ and $B$, and hence by Theorem 2.7 the homomorphisms $\phi_{A}: A \rightarrow A / X$, $\phi_{B}: B \rightarrow B / Y$ can be extended to a homomorphism $\phi$ of $F$ onto $\bar{F}$. Then

$$
H \phi=H \phi_{A}=H X / X \cong H /(H \cap X)=H / N .
$$

Similarly $A \phi=A / X$ and $B \phi=B / Y$.

Now let $x \in H \cap[A, B]$. Then $x \phi \in(H \cap[A, B]) \phi \leqq H \phi \cap[A \phi, B \phi]$ (Lemma 2.3) $=H / N \cap[A / X, B / Y]$. But $H / N \cong H X / X$ is central in $A / X$ and similarly in $B / Y$. Thus, by Lemma 2.9, $H / N \cap[A / X, B / Y]=E$ and hence $H \cap[A, B]$ is contained in $\operatorname{ker} \phi$ (the kernel of $\phi$ ). It follows that $H \cap[A, B] \leqq H \cap \operatorname{ker} \phi$. However, $H \cap \operatorname{ker} \phi=H \cap \operatorname{ker} \phi_{A}=H \cap X=N$. Hence $H \cap[A, B] \leqq N$. Since the reverse inequality follows immediately from the definition of $N$, Lemma 3.1 is proved.

Note 3.2. The above result extends to amalgams of any number of groups provided there remains just one amalgamated subgroup. The only change in the above proof occurs in the last paragraph where (calling the constitutent groups $A_{\alpha}(\alpha \in I)$ ) we should have to take $x \in H \cap\left[A_{\alpha}\right]^{F}$ rather than $x \in H \cap\left[A_{\alpha}\right]$. However, Lemma 2.3 and Lemma 2.9 are still sufficient to deal with this and the required extension follows.

We momentarily leave the proof of the theorem to obtain a lemma (Lemma 3.4) which, although it is not used in the proof of the theorem, is the converse of Lemma 3.7 which is an essential part of the proof. Together Lemmas 3.4 and 3.7 extend (and complete) theorems given by the author in [1].

First we have

LEMMA 3.3. Let $G$ be a generalised regular product of its subgroups $A_{\alpha}(\alpha \in I)$ with the single amalgamated subgroup $H$. Then, in $G$,

$$
H \cap\left[A_{\alpha}\right]^{G}=\prod_{\alpha \in I}\left(H \cap\left[A_{\alpha}, H\right]\right) .
$$


Proof. To avoid confusion let $F$ be the generalised free product of groups $\hat{A}_{\alpha}$ amalgamating the subgroup $\hat{H}$ where $\hat{A}_{\alpha} \cong A_{\alpha}$ and $\hat{H} \cong H$. Note 3.2 tells us that the equality

$$
\hat{H} \cap\left[\hat{A}_{\alpha}\right]^{F}=\prod_{\alpha \in I}\left(\hat{H} \cap\left[\hat{A}_{\alpha}, \hat{H}\right]\right)
$$

holds. If we let $\phi$ denote the natural homomorphism from $F$ to $G$ we have by Lemma 2.11, since $\operatorname{ker} \phi \leqq\left[\hat{A}_{\alpha}\right]^{F}$,

$$
\begin{aligned}
H \cap\left[A_{\alpha}\right]^{G} & =\hat{H} \phi \cap\left[\hat{A}_{\alpha} \phi\right]^{F \phi}=\left(\hat{H} \cap\left[\hat{A}_{\alpha}\right]^{F}\right) \phi \\
& =\left(\prod_{\alpha \in I}\left(\hat{H} \cap\left[\hat{A}_{\alpha}, \hat{H}\right]\right)\right) \phi=\prod_{\alpha \in I}\left\{\left(\hat{H} \cap\left[\hat{A}_{\alpha}, \hat{H}\right]\right) \phi\right\} \\
& \leqq \prod_{\alpha \in I}\left(\hat{H} \phi \cap\left[\hat{A}_{\alpha}, \hat{H}\right] \phi\right)=\prod_{\alpha \in I}\left(H \cap\left[A_{\alpha}, H\right]\right) .
\end{aligned}
$$

Thus $H \cap\left[A_{\alpha}\right]^{G} \leqq \prod_{\alpha \in I}\left(H \cap\left[A_{\alpha}, H\right]\right)$ in $G$ and, since the reverse inequality clearly holds, the lemma is proved.

Lemma 3.4. Let $G$ be a generalised regular product of its subgroups $A_{\alpha}(\alpha \in I$, $I$ assumed ordered) with the single amalgamated subgroup $H$. Choose in each $A_{\alpha}$ a set $S_{\alpha}$ of left coset representatives (including $\left.1_{\alpha}\right)$ of $A_{\alpha}$ modulo $H^{A_{\alpha}}\left(=H\left[H, A_{\alpha}\right]\right)$ and let $Q$ be a system of left coset representatives (including $1_{H}$ ) of $H$ modulo $\prod_{\alpha \in I}\left(H \cap\left[A_{\alpha}, H\right]\right)$. Then every (nonidentity) element $g$ of $G$ can be written uniquely in the form $g=s_{1} s_{2} \cdots s_{m} q u$ where $1 \neq s_{i} \in S_{\alpha_{i}}, q \in Q, u \in\left[A_{\alpha}\right]^{G}$ and $\alpha_{1}<\alpha_{2}<\cdots<\alpha_{m}$.

Proof. Since $G$ is generated by the $A_{\alpha}$ it is easy to see that every element $g$ of $G$ can be written as

$$
g=a_{1} \cdots a_{m} v
$$

where $a_{i} \in A_{\alpha_{i}}, v \in\left[A_{\alpha}\right]^{G}$ and $\alpha_{1}<\alpha_{2}<\cdots<\alpha_{m}$. We then put $a_{1}=s_{1} h_{1} h_{\alpha_{1}}$ where $s_{1} \in S_{\alpha_{1}}, h_{1} \in H$ and $h_{\alpha_{1}} \in\left[A_{\alpha_{1}}, H\right]$. Thus, since $h_{\alpha_{1}}$ and hence any conjugate of it belongs to $\left[A_{\alpha}\right]^{G}$, we can write $g=s_{1}\left(h_{1} a_{2}\right) a_{3} \ldots a_{m} v_{1}$ where $v_{1} \in\left[A_{\alpha}\right]^{G}$. Now $h_{1} a_{2} \in A_{\alpha_{2}}$ and we repeat the process above. After $m$ steps we obtain $g=s_{1} \cdots s_{m} h_{m} v_{m}$ where $h_{m} \in H, v_{m} \in\left[A_{\alpha}\right]^{G}$. We now write $h_{m}=q v_{m+1}$ where $q \in Q, v_{m+1} \in\left[A_{\alpha}\right]^{G}$, and putting $u=v_{m+1} v_{m}$ we see that $g=s_{1} \cdots s_{m} q u$ as required.

To prove uniqueness suppose that

$$
g=s_{1} \cdots s_{m} q u=s_{1}^{\prime} \cdots s_{n}^{\prime} q^{\prime} u^{\prime}
$$

where $s_{i}^{\prime} \in A_{\beta_{1}}, q^{\prime} \in Q, u^{\prime} \in\left[A_{\alpha}\right]^{G}$ and $\beta_{1}<\beta_{2}<\cdots<\beta_{n}$. If $\alpha_{1}<\beta_{1}$, say, then using Lemma 2.10, $s_{1}=s_{1}^{\prime} \cdots s_{n}^{\prime} q^{\prime} u^{\prime}\left(s_{2} \cdots s_{m} q u\right)^{-1} \in A_{\alpha_{1}} \cap\left\{\alpha_{1} A\right\}^{G}=H^{A_{\alpha_{1}}}$. Hence $s_{1} \in H^{A_{\alpha_{1}}}$ so that $s_{1}=1$. Similarly, if $\alpha_{1}=\beta_{1}$ we obtain $\left(s_{1}^{\prime}\right)^{-1} s_{1} \in H^{A_{\alpha_{1}}}$ which implies $s_{1}=s_{1}^{\prime}$. In this case we cancel $s_{1}$ and $s_{1}^{\prime}$ in (3.6) and repeat the process with the new equality. It is thus clear that we must have $m=n$ and $s_{i}=s_{i}^{\prime}(i=1, \ldots, m)$. This leaves

$$
q u=q^{\prime} u^{\prime} \text { or } \quad\left(q^{-1}\right) q^{\prime}=u\left(u^{\prime}\right)^{-1} \in H \cap\left[A_{\alpha}\right]^{G} .
$$


By Lemma 3.3 and the definition of $Q$ this implies $q=q^{\prime}$ and hence $u=u^{\prime}$ so that the uniqueness part of the theorem is proved. Conversely, we have

LEMMA 3.7. Let $G$ be a group generated by its subgroups $A_{\alpha}(\alpha \in I, I$ assumed ordered) with the single amalgamated subgroup $H$. Let $S_{\alpha}$ and $Q$ be as above and suppose that every element of $G$ can be expressed uniquely in the form $g=s_{1} s_{2} \cdots s_{m} q u$ where $s_{i} \in S_{\alpha_{i}}, q \in Q, u \in\left[A_{\alpha}\right]^{G}$ and $a_{1}<\cdots<\alpha_{m}$. Then $G$ is a generalised regular product of the $A_{\alpha}$ amalgamating $H$.

Proof. As in Lemma 3.3 we take isomorphic copies $\hat{A}_{\alpha}$ and $\hat{H}$ of $A_{\alpha}$ and $H$ respectively and let $F$ be the free product of the $\hat{A}_{\alpha}$ amalgamating $\hat{H}$. If $\phi$ is the natural homomorphism from $F$ onto $G$ we must show that $\operatorname{ker} \phi \leqq\left[\hat{A}_{\alpha}\right]^{F}$.

We choose in each $\hat{A}_{\alpha}$ a system of left coset representatives $\hat{S}_{\alpha}$ taken modulo $\hat{H}\left[\hat{H}, \hat{A}_{\alpha}\right]$ as follows. If $s_{\alpha} \in S_{\alpha}$, we choose $\hat{s}_{\alpha}$ to be that element of $\hat{A}_{\alpha}$ which is mapped under $\phi$ onto $s_{\alpha}$. ( $\phi$ is an isomorphism on $\hat{A}_{\alpha}$ and so $\hat{s}_{\alpha}$ is uniquely determined.) In a similar manner we choose a set of left coset representatives $\hat{Q}$ of $\hat{H}$ modulo $\prod_{\alpha \in I}\left(\hat{H} \cap\left[\hat{A}_{\alpha}, \hat{H}\right]\right)$.

Since $F$ is a generalised regular product of the $\hat{A}_{\alpha}$, we may write, using Lemma 3.4, any $f \in F$ in the form

$$
f=\hat{s}_{1} \hat{s}_{2} \cdots \hat{s}_{m} \hat{q} \hat{u}
$$

If $f \in \operatorname{ker} \phi$, then

$$
1=f \phi=\hat{s}_{1} \phi \cdot \hat{s}_{2} \phi \cdots \hat{s}_{m} \phi \cdot \hat{q} \phi \cdot \hat{u} \phi .
$$

But, by choice of $\hat{S}_{\alpha}$ and $\hat{Q}, \hat{s}_{i} \phi \in S_{\alpha_{i}}$ and $\hat{q} \phi \in Q$. Further $\hat{u} \phi \in\left[\hat{A}_{\alpha}\right]^{F} \phi=\left[A_{\alpha}\right]^{G}$. Our hypothesis then implies that

$$
\hat{s}_{1} \phi=\hat{s}_{2} \phi=\cdots=\hat{s}_{m} \phi=\hat{q} \phi=\hat{u} \phi=1 .
$$

As $\phi$ is an isomorphism on the $\hat{A}_{\alpha},(3.8)$ leads to

$$
\hat{s}_{1}=\hat{s}_{2}=\cdots=\hat{s}_{m}=\hat{q}=1
$$

from which $f=\hat{u} \in\left[\hat{A}_{\alpha}\right]^{F}$ follows.

We can now give the

Proof of the theorem. Let $P$ be the permutational product of $(A, B ; H)$ with the systems $\bar{S}, \bar{T}$ of left coset representatives and let $p \in P$. Since $P$ is generated by $\rho(A), \rho(B)$ it is easy to see that $p$ can be written in the form $p=\rho(a) \rho(b) U$ where $U \in[\rho(A), \rho(B)]$. In fact, if we select systems $S, T, Q$ of left coset representatives (each including the unit element) of $\rho(A)$ modulo $\rho\left(H^{A}\right)$, of $\rho(B)$ modulo $\rho\left(H^{B}\right)$, and of $\rho(H)$ modulo $\rho((H \cap[A, H])(H \cap[H, B]))$, respectively, we can show, as in Lemma 3.4, that $p$ may be written

$$
p=\rho(s) \rho(t) \rho(q) U
$$

where $\rho(s) \in S, \rho(t) \in T, \rho(q) \in Q$, and $U \in[\rho(A), \rho(B)]$. 
We now show that if $p=1$, then $\rho(s)=\rho(t)=\rho(q)=U=1$. By Lemma 3.7 this will be sufficient to prove the theorem.

We first note that $U$ is a product of elements of the form $[\rho(a), \rho(b)]$ and their inverses where $\rho(a) \in \rho(A), \rho(b) \in \rho(B)$. We can therefore write

$$
U=\prod_{i=1}^{n}\left[\rho\left(a_{2 i-1}\right), \rho\left(b_{2 i-1}\right)\right]\left[\rho\left(b_{2 i}\right), \rho\left(a_{2 i}\right)\right]
$$

where we may have to take some of these commutators as the unit element. For ease in the proof we shall assume $U=[\rho(a), \rho(b)]$; the general case is just as straightforward.

Suppose, then, that $p=\rho(s) \rho(t) \rho(q) U=1$ where $U=[\rho(a), \rho(b)]$. Selecting an arbitrary $\sigma \in \bar{S}, \tau \in \bar{T}$ and $1 \in H$, we look at the image of the triplet $(\sigma, \tau, 1)$ under $p$. We have

$$
\begin{aligned}
& (\sigma, \tau, 1)^{\rho(s)}=\left(\sigma_{1}, \tau, k_{1}\right) \quad \text { where } \sigma_{1} k_{1}=\sigma s, \\
& \left(\sigma_{1}, \tau, k_{1}\right)^{\rho(t)}=\left(\sigma_{1}, \tau_{1}, k_{2}\right) \text { where } \tau_{1} k_{2}=\tau k_{1} t, \\
& \left(\sigma_{1}, \tau_{1}, k_{2}\right)^{\rho(q)}=\left(\sigma_{1}, \tau_{1}, k\right) \text { where } k=k_{2} q \text {, } \\
& \left(\sigma_{1}, \tau_{1}, k\right)^{\rho(a-1)}=\left(s_{1}, \tau_{1}, h_{1}\right) \text { where } s_{1} h_{1}=\sigma_{1} k a^{-1} \text {, } \\
& \left(s_{1}, \tau_{1}, h_{1}\right)^{\rho(b-1)}=\left(s_{1}, t_{1}, h_{2}\right) \quad \text { where } t_{1} h_{2}=\tau_{1} h_{1} b^{-1} \text {, } \\
& \left(s_{1}, t_{1}, h_{2}\right)^{\rho(a)}=\left(s_{2}, t_{1}, h_{3}\right) \quad \text { where } s_{2} h_{3}=s_{1} h_{2} a \text {, } \\
& \left(s_{2}, t_{1}, h_{3}\right)^{\rho(b)}=\left(s_{2}, t_{2}, h_{4}\right) \quad \text { where } \quad t_{2} h_{4}=t_{1} h_{3} b \text {. }
\end{aligned}
$$

It follows that

$$
\begin{aligned}
& s_{2}=s_{1} h_{2} a h_{3}^{-1}=\sigma_{1} k a^{-1} h_{1}^{-1} h_{2} a h_{3}^{-1}=\sigma s k_{1}^{-1} k a^{-1} h_{1}^{-1} h_{2} a h_{3}^{-1}, \\
& t_{2}=t_{1} h_{3} b h_{4}^{-1}=\tau_{1} h_{1} b^{-1} h_{2}^{-1} h_{3} b h_{4}^{-1}=\tau k_{1} t k_{2}^{-1} h_{1} b^{-1} h_{2}^{-1} h_{3} b h_{4}^{-1} .
\end{aligned}
$$

But $p=1$ and so $s_{2}=\sigma, t_{2}=\tau, h_{4}=1$. Thus from (3.9)

$$
\begin{array}{ll}
1=s k_{1}^{-1} k \cdot h_{1}^{-1} h_{2} h_{3}^{-1} \cdot x & \text { where } x \in[A, H] ; \\
1=k_{1} t k_{2}^{-1} \cdot h_{1} h_{2}^{-1} h_{3} \cdot y & \text { where } y \in[B, H] .
\end{array}
$$

From (3.10) it follows that $s \in H[H, A]=H^{A}$, and hence that $s=1$. This, in turn, implies that $x \in[A, H] \cap H$. Similarly, $t \in H^{B}$ so that $t=1$ and $y \in[B, H] \cap H$. Finally, we rewrite the second equality of (3.10) as

$$
\begin{aligned}
1 & =k_{1} \cdot q k^{-1} \cdot h_{1} h_{2}^{-1} h_{3} y=q k_{1}\left[k_{1}, q\right] k^{-1} \cdot h_{1} h_{2}^{-1} h_{3} y \\
& =q k_{1} k^{-1} \cdot h_{1} h_{2}^{-1} h_{3} \cdot y^{*}
\end{aligned}
$$

where $y^{*} \in[B, H] \cap H .(3.10)$ and (3.11) then yield

$$
q^{-1} \cdot 1=k_{1} k^{-1} \cdot h_{1} h_{2}^{-1} h_{3} y^{*} \cdot k_{1}^{-1} k \cdot h_{1}^{-1} h_{2} h_{3}^{-1} \cdot x .
$$

It is now clear that $q^{-1} \in(H \cap[A, H])(H \cap[H, B])$. But by choice of $Q$ this means that $q=1$. We have thus shown that $s=t=q=1$. This implies that $\rho(s)=\rho(t)$ $=\rho(q)=1$ and hence that $U=1$. This completes the proof of the theorem. 


\section{REFERENCES}

1. R. B. J. T. Allenby, Normal forms for generalised regular products of groups, Math. Z. 102 (1967), 356-369.

2. O. N. Golovin, Nilpotent products of groups, Mat. Sb. 27 (69) (1950), 427-454; English transl., Amer. Math. Soc. Transl. (2) 2 (1956), 89-115.

3. R. J. Gregorac, Permutational products and the embedding theory of group amalgams, J. Austral. Math. Soc. (to appear).

4. G. Higman, Amalgams of p-groups, J. Algebra (3) 1 (1964), 301-305.

5. S. Moran, Associative operations on groups. III, Proc. London Math. Soc. (3) 9 (1959), 287-317.

6. B. H. Neumann, Permutational products of groups, J. Austral. Math. Soc. 1 (1960), 299310.

7. - - An essay on free products of groups with amalgamations, Philos. Trans. Roy. Soc. London Ser. A 246 (1954), 503-554.

8. J. Wiegold, Some remarks on generalised products of groups with amalgamations, Math. $Z$. 75 (1961), 57-78.

THE UNIVERSITY,

LEEDS, ENGLAND 